\title{
La eficacia de la web corporativa en las pequeñas y medianas empresas: un análisis de la usabilidad web
}

\author{
Pedro Pablo Marín DUEÑAS \\ Universidad de Cádiz \\ pablo.marin@uca.es
Carmen LASSO de LA Vega GonZÁleZ
Universidad de Cádiz
carmen.lasso@uca.es \\ Juan José Mier-TerÁN Franco \\ Universidad de Cádiz \\ juanjose.mier-teran@uca.es
}

Recibido: 14 de mayo de 2015

Aceptado: 8 de octubre de 2015

\section{Resumen}

Internet como un nuevo medio y la web como su principal exponente han transformado la manera en que las empresas se comunican con sus públicos, favoreciendo las relaciones entre éstos y aportando notables beneficios para las organizaciones. Esta importancia ha llevado a las pequeñas y medianas empresas (PYMES) a desarrollar sites corporativos para dar a conocer sus negocios. Este trabajo, aplicando la metodología del análisis de contenido, analiza los principales factores y herramientas que hacen de las Webs sitios usables e intuitivos que fomenten unas mejores relaciones entre las PYMES y sus públicos. Además, se ha elaborado un índice para medir la eficacia de dichas Webs desde la perspectiva de la usabilidad. Los resultados indican que las Webs de las PYMES tienen, por lo general, una usabilidad aceptable y poseen unos índices de navegabilidad adecuados.

Palabras clave: Web, pequeñas y medianas empresas, comunicación empresarial, usabilidad, eficacia.

\section{The effectiveness of corporate Web in small and médium-sized business: an analysis of Web Usability}

\begin{abstract}
Internet and the Web have changed the way that companies communicate with their publics, improving relations between them. Also providing substantial benefits for organizations. This has led to small and medium enterprises (SMEs) to develop corporate sites to establish relationships with their audiences. This paper, applying the methodology of content analysis, analyzes the main factors and tools that make the Websites usable and intuitive sites that promote better relations between SMEs and their audiences. Also, it has developed an index to measure the effectiveness of Webs from the perspective of usability. The results indicate that the Websites have, in general, appropriate levels of usability.

Keywords: Website, small and medium-sized business, business communication, usability, effectiveness.

\section{Referencia normalizada}

MARÍN DUEÑAS, Pedro Pablo; LASSO DE LA VEGA GONZÁLEZ, Carmen; y MIER-TERÁN FRANCO, Juan José (2016): "La eficacia de la web corporativa en las pequeñas y medianas empresas: un análisis de la usabilidad web". Estudios sobre el Mensaje Periodístico. Vol. 22, Núm. 1 (enero-junio), págs.: 431-444. Madrid, Ediciones Complutense.
\end{abstract}

Sumario: 1. Introducción. 2. La usabilidad web. 3. Metodología; 3.1. Muestra; 3.2. Procedimiento y método. 4. Análisis y resultados; 4.1. Identificación, compatibilidad, rotulado, mapa web y actualización; 4.2. Lenguaje y redacción; 4.3. Diseño de la página; 4.4. Elementos multimedia; 4.5. Existencia de buscadores. 5. Conclusiones. 6. Referencias bibliográficas. 


\section{Introducción}

Las Nuevas Tecnologías de la Información y la Comunicación (NTICS), como Internet, con la web como máximo exponente, han transformado las formas de comunicación entre las empresas y sus públicos, proporcionando beneficios y ofreciendo nuevos modos, nuevos caminos y nuevas e innovadoras formas de interacción e intercambio de mensajes (Milojevic, Kleut y Ninkovic, 2013) para todos los sujetos implicados en el proceso comunicativo. Un proceso que no sólo ha revolucionado las bases de la publicidad convencional, sino que propone un cambio de paradigma (Bautista, 2012) en la comunicación interpersonal, colectiva y masiva.

La web puede ayudar a las empresas a lograr una mayor visibilidad, incrementando así sus beneficios, llegar a nuevos mercados, mejorar sus servicios al cliente, transferir sus productos/servicios de una forma más eficiente, trasladar su identidad a la sociedad, favoreciendo la consecución de una imagen positiva y lo que ello conlleva, dada la importancia para las organizaciones en general del conocimiento y reconocimiento de sus marcas, así como para las pequeñas y medianas empresas en particular, por su gran peso en el tejido empresarial de la mayoría de los países desarrollados.

Internet se ha constituido más que como un nuevo medio de comunicación, en un recurso ineludible para que las empresas puedan gestionar y lograr sus objetivos y, dada la importancia que ha alcanzado, actualmente resulta imprescindible su aplicación por parte de las compañías debido, entre otras cosas, a la rentabilidad que genera una oportuna interacción comunicativa entre la empresa u organización y sus públicos objetivos. Se hace necesaria la implantación de páginas web que favorezcan estas relaciones, produciendo una necesidad que haga que el usuario vuelva a la web ( $\mathrm{Pa}-$ lazón, 2001).

El notable desarrollo de la tecnología web hace plantearse a los directivos, gerentes y propietarios de las pequeñas y medianas empresas, la necesidad de comunicar sus negocios a través del portal corporativo. Y esta importancia ha llevado a la proliferación, lenta pero constante, de PYMES que cuentan con página web.

Debe tenerse en cuenta, en este sentido, que la pequeña y mediana empresa se ha caracterizado por el empleo, intuitivo y no planificado, de técnicas de Comunicación interpersonal y colectiva, coloquialmente conocidas como "boca-oído" y entendidas, en este ámbito comunicativo-empresarial, como las más adecuadas para su promoción, de manera independiente a los diversos avances en las estrategias comunicativas y sus emergentes formas de implementación. Esto es, la pequeña empresa española no contemplaba la necesidad de la inversión en Comunicación. Una percepción que ha cambiado de manera casi radical y en muy poco tiempo, con la consolidación del medio Internet como el más importante y rentable recurso de Comunicación persuasiva, informativa y de ocio, que debe ser entendido desde esta vertiente tripartita con el fin de operar una gestión comunicativa eficaz y rentable, ya que no sólo se trata de aparecer en la red de redes, sino que debe tratarse de una aparición prefijada y controlada, con el objeto de minimizar costes y recursos y de maximizar beneficios.

De ahí que, ante la relevancia que este medio está adquiriendo para la Comunicación de las empresas, se ha fijado como objetivo de esta investigación el análisis de portales web de pequeñas y medianas empresas, desde el punto de vista de la usabili- 
dad que las mismas ofrecen a la hora de facilitar las relaciones de estas organizaciones con sus públicos de interés. Tal y como apuntan Da Silva y Alwi (2005) o Huertas y Xifra (2009) la usabilidad es una característica intrínseca de las páginas web y debe ser el factor clave en el diseño de estrategias de comunicación empresarial a través de la WWW (Garcia et al., 2012).

Se han estudiado, a partir de la metodología del análisis de contenido, los principales factores y herramientas que hacen de las webs sitios usables e intuitivos que fomenten unas mejores relaciones entre las PYMES y sus públicos. Además, se ha elaborado un índice para medir la eficacia de dichas webs desde la perspectiva de la usabilidad como aspecto estratégico en el diseño de la Comunicación empresarial digital.

\section{La usabilidad web}

La llegada de Internet ha hecho cambiar las estrategias de comunicación y de marca de la mayoría de empresas que, aunque careciesen de ella, empiezan a preocuparse por las mismas (García y Castillo, 2010). La expansión de Internet ha supuesto la creciente capacidad para las empresas de expandir su actividad, permitiendo una relación más personalizada con sus públicos. Gibson (2000) señala la capacidad de las webs corporativas para mantener relaciones interactivas con los públicos convirtiéndose en uno de sus principales medios de relación con sus distintos públicos objetivo ofreciendo numerosas ventajas para las empresas.

La web se ha convertido en uno de los medios de comunicación con mayor atención por parte de las empresas que se lanzan a la conquista de la misma no sólo como un medio de generación de ingresos, sino como una fuente para la difusión de la información y la comunicación (Alamdari y Mason, 2006). El portal digital de una empresa pasa a ser una herramienta imprescindible para todas las personas que se relacionan con ella configurándose, a su vez, como un instrumento básico para las organizaciones proporcionando una serie de oportunidades y beneficios y permitiendo alcanzar ventajas competitivas (García, 2012; Ros, 2008; Díaz, 2008; Wang and Fesenmaier, 2006; Xing and Grandt, 2006; Yoon et al., 2006).

La necesidad de diferenciar la empresa hace necesaria una estrategia de comunicación e imagen corporativa en la web, que ponga de manifiesto los esfuerzos de las empresas por integrar a sus públicos en las mismas. Las instituciones empresariales necesitan tener una presencia virtual eficaz para propiciar y fomentar la comunicación con esos públicos. Y, en este sentido, se puede afirmar que las variables que favorecen esa eficacia comunicativa entre la empresa y los usuarios de una página web son, fundamentalmente la usabilidad, la interactividad y el contenido (Kaplanidou and Vogt, 2006; Ha \& Love, 2005; Kline et al., 2004; Morrison et al.).

El concepto de usabilidad (que incluye la accesibilidad o navegabilidad de la página web) está directamente relacionado con la satisfacción del usuario (Hassan, 2006) ya que el diseño de un sitio web puede convertirse en un primer obstáculo entre éste (como receptor del mensaje) y la fuente de información. Por tanto, la usabilidad se configura como un elemento básico para una comunicación eficaz de una página web. Se ha considerado que una página web que cuenta con usabilidad proporciona al usua- 
rio la información necesaria de una forma accesible. En sentido contrario, problemas en la usabilidad del site pueden provocar que el usuario de por terminada la navegación por la página web (Sutcliffe, 2002).

Krug (2006) ofrece una definición sobre el término usabilidad según la cual la usabilidad no es más que estar seguro de que algo funciona bien. Es decir, que una persona con habilidades medias pueda utilizar una cosa con un fin concreto sin terminar frustrado. Entendida la usabilidad, en definitiva, como el grado de facilidad en el uso de los distintos elementos de un documento web, puede ser definida a partir de 5 atributos (Nielsen, 1993):

- Facilidad de aprendizaje.

- Eficiencia.

- Retención sobre el tiempo. El uso del sitio, no debe olvidarse pasado un tiempo desde su aprendizaje.

- Minimizar los errores e indicar al usuario cómo solucionarlos en el caso de que se produzcan.

- Satisfacción subjetiva. ¿Les gusta el uso del sistema a los usuarios?

Hassan (2006) considera que la usabilidad debe ser un factor transversal en el diseño de una página web y que debe orientarse al usuario y a su no frustración, dotando a la misma de funcionalidad y proporcionando, además, un factor motivador. La usabilidad debe ser un componente determinante en la interacción del usuario con la web (Li et al., 2006) posibilitando que la empresa se presente correctamente ante sus públicos (Pollach, 2005) e influyendo de manera positiva en la navegación del usuario. La usabilidad que un sitio web debe ofrecer a sus usuarios, abarca cuatro objetivos principales (Hernández, Jiménez y Martín, 2008):

- Organizar y clasificar contenido.

- Etiquetar información.

- Diseñar sistemas de navegación.

- Ayudar a los usuarios a encontrar información.

En definitiva, se hace necesario integrar la usabilidad como un aspecto más de la correcta comunicación con los públicos de las empresas para poder transmitir así una imagen fuerte y sólida que permita a los usuarios acceder y manejar fácilmente los mensajes que las empresas trasmiten a través de sus webs (García y Castillo, 2010).

\section{Metodología}

Se ha elegido el análisis de contenido como técnica para estudiar las sedes webs corporativas de las PYMES, por tratarse de una herramienta centrada en la identificación, codificación y categorización de información, de forma que el investigador sea capaz de conocer el objeto de estudio de forma objetiva y sistemática (García, 2012).

Particularmente, esta técnica resulta muy utilizada por su rentabilidad y alto grado de facilidad de sistematización de uno o varios enfoques determinados, empleado con asiduidad en casi todos los ámbitos de la investigación científica en Comunicación. Es decir, que es un método bastante recurrido y eficaz a la hora de analizar los conteni- 
dos mediáticos a través de aplicaciones concretas (Villena, 2013). Concretamente, el análisis de contenido ha sido validado en estudios anteriores, demostrando su eficacia para conocer la realidad de una organización a través de sus sedes webs.

\subsection{Muestra}

Para aplicar la metodología del análisis de contenido que permita el estudio de la usabilidad web se ha seleccionado una muestra aleatoria compuesta por 130 páginas web de empresas ubicadas en la provincia de Cádiz. Dicha muestra ha sido extraída de la base de datos SABI, que en el mes de julio de 2014 incluía 2373 empresas situadas en la región y con sede web activa.

\subsection{Procedimiento y método}

Piñuel (2002) identifica una serie de pasos que son necesarios a la hora de ejecutar un análisis de contenido y que se establecen como el procedimiento desarrollado en el proceso investigador

1. Selección de la comunicación que será estudiada. En este caso será la comunicación que las pequeñas y medianas empresas realizan a través de sus sites corporativos.

2. Selección de las categorías que se utilizarán. Las categorías son los aspectos genéricos que serán evaluados. Se trata de establecer una serie de grandes bloques sobre los que se realizará el análisis y los cuales serán desarrollados en indicadores concretos que dan la información necesaria para cada uno de estos grupos. La categoría de análisis será la usabilidad de las páginas webs de la muestra seleccionada

3. Selección de las unidades de análisis. Los indicadores son los elementos que desarrollan cada uno de las categorías establecidas para el análisis de la información. Para la selección de estos ítems de medida se han tomado como referencia escalas de medida ya utilizadas en trabajos previos (fuentes secundarias), que han sido adaptadas al contexto específico de la presente investigación, así como algunos ítems de elaboración propia y a partir de las cuales se ha diseñado la plantilla de análisis. Concretamente se han seleccionado 25 ítems para medir la usabilidad, a partir de la revisión de los siguientes trabajos: Nielsen y Tahir (2002); Palmer (2002); Juliá, García y Polo (2004); Moreno y Capriotti (2006); Hernández, Jiménez y Martín (2008); Díaz, Martín y Esteban (2008); Xifra y Huertas (2009); Castillo, Carrillo y Luna (2012); García, Carrillo y Durán (2012); Gómez, Tapia y Díaz (2012).

4. Selección del sistema de recuento o de medida. En el caso concreto de esta investigación se ha optado por seguir un sistema de valoración basado en los trabajos de Castillo, Carrillo y Luna (2012), por un lado, y de Nielsen y Tahir (2002), por otro.

Así, el sistema de puntuación empleado ha sido el siguiente:

- Mala gestión o inexistencia/no presencia del indicador: 0 puntos

- El indicador se aprecia de forma modesta: 1 punto

- El indicador se aprecia de forma notable: 2 puntos

- Buena gestión o existencia/presencia del indicador: 3 puntos 
Finalmente se llevó a cabo la tabulación y depuración de los datos así como el análisis estadístico de los mismos. Para el análisis de los datos y de cara al tratamiento estadístico de los mismos se ha utilizado la herramienta estadística SPSS.

\section{Análisis y resultados}

\subsection{Identificación, compatibilidad, rotulado, mapa web y actualización}

La web está perfectamente identificada a partir de la dirección URL. De hecho, en la mayoría de los casos, la URL coincide totalmente con el nombre de la empresa, lo que facilita enormemente su recuerdo. Además, permite transmitir una única marca y un único nombre en cualquier soporte en el que se realice una acción de Comunicación.

En un plano más técnico, ofrecen, en general, una adecuada compatibilidad con los distintos navegadores que ofrece el mercado.

Respecto al menú de acceso a las secciones o rotulado este indicador ofrece resultados divergentes. La mitad de las webs cuentan con un menú permanente que se mantiene presente en cualquier sección de la webs facilitando la navegación por la misma.

$\mathrm{La}$ arquitectura web de la página supone un elemento clave para que el usuario acceda a la información sin problemas y sea capaz de visitar la página con comodidad. Identificar cada sección con un rótulo convencional y familiar es un elemento imprescindible, ofreciendo al usuario información en secciones que éste pueda identificar de un solo vistazo.

En el caso de las PYMES estudiadas, el rotulado cobra especial importancia, puesto que la mayoría de ellas disponen de webs bastante sencillas. En ellas, los contenidos ofrecidos se presentan en estas secciones, ocupando un lugar destacado en la interfaz principal que permite al usuario identificar las secciones principales de la web y acceder a la información sobre la empresa o sobre sus productos/servicios. Esta estructura facilita al usuario localizar la información deseada, transmitiendo una sensación de sencillez y claridad.

En cuanto a la existencia de mapa web, es decir, la disposición de un mapa del sitio web para facilitar a los usuarios de una manera más eficiente la visualización de la disposición de los contenidos incorporados y que sirve de guía para el usuario apenas está presente en las webs de las pequeñas y medianas empresas debido, en gran medida, a la sencillez en cuanto al diseño de la mayoría de las webs de las pequeñas y medianas empresas.

Analizada la actualización de las webs, las PYMES, en general, gestionan este parámetro de una forma correcta, actualizando los contenidos que ofrecen en sus webs ( $60 \%$ de los casos). Este factor da credibilidad a la web, pues el usuario percibe que las PYMES tienen interés y prestan atención a sus sites corporativos y no son meros elementos decorativos y es que, la falta de revisión frecuente de los contenidos y estructura de una web puede denotar falta de preocupación de la empresa y, en consecuencia, una falta de credibilidad que podría perjudicar la imagen de la empresa y es que un sitio web debería contener información completa, exacta y actualizada para todos los usuarios interesados por ella. 
Tabla 1. Identificación, compatibilidad, rotulado, mapa web y actualización en las webs.

Fuente: elaboración propia

\begin{tabular}{|l|c|c|c|c|c|}
\hline \multicolumn{1}{|c|}{$\begin{array}{c}\text { Unidades de } \\
\text { análisis }\end{array}$} & $\begin{array}{c}\text { URL } \\
(\%)\end{array}$ & $\begin{array}{c}\text { Compatibilidad } \\
(\mathbf{\%})\end{array}$ & $\begin{array}{c}\text { Rotulado } \\
(\mathbf{\%})\end{array}$ & $\begin{array}{c}\text { Mapa Web } \\
(\mathbf{\%})\end{array}$ & $\begin{array}{c}\text { Actualización } \\
(\mathbf{\%})\end{array}$ \\
\hline $\begin{array}{l}\text { Mala gestión } \\
\text { inexistencia del } \\
\text { indicador }\end{array}$ & $18(14)$ & $39(30)$ & $63(48)$ & $102(78)$ & $51(40)$ \\
\hline $\begin{array}{l}\text { Se aprecia de } \\
\text { forma muy } \\
\text { modesta }\end{array}$ & 0 & 0 & 0 & 0 & 0 \\
\hline $\begin{array}{l}\text { Se aprecia de } \\
\text { forma más notable }\end{array}$ & 0 & $20(16)$ & 0 & 0 & $27(27)$ \\
\hline $\begin{array}{l}\text { Buena } \\
\text { gestión/existencia } \\
\text { del indicador }\end{array}$ & $\begin{array}{c}112 \\
(86)\end{array}$ & $71(54)$ & $67(52)$ & $28(22)$ & $52(40)$ \\
\hline
\end{tabular}

\subsection{Lenguaje y redacción}

- Página web en más de un idioma. Acceso a la información en varios idiomas.

- Emplea un lenguaje claro y conciso.

- Utiliza correctamente las mayúsculas y otras reglas de corrección ortográfica.

- Correcta utilización de estilo y otras cuestiones redaccionales.

- Se ha evitado la sobrecarga informativa.

En general, la gestión de los indicadores relacionados con el lenguaje y la redacción están bien tratados en las webs. Tanto la corrección ortográfica, como la claridad y la sencillez del lenguaje empleado ofrecen datos positivos. Lo mismo ocurre con las cuestiones de estilo y la gestión de la información que se ofrece. Las PYMES eligen bien el lenguaje utilizado para comunicarse con sus públicos $\mathrm{y}$, aunque no ofrecen grandes cantidades de información, esta falta de densidad informativa facilita la mejor transmisión de los mensajes, que llegan al usuario mediante un lenguaje familiar y cercano.

Tabla 2. Lenguaje y redacción en la web. Fuente: elaboración propia

\begin{tabular}{|l|c|c|c|c|c|}
\hline Unidades de análisis & Idiomas & $\begin{array}{l}\text { Lenguaje } \\
\text { claro }\end{array}$ & Ortografía & $\begin{array}{l}\text { Redacción } \\
\text { y estilo }\end{array}$ & $\begin{array}{l}\text { Sobrecarga } \\
\text { informativa }\end{array}$ \\
\hline $\begin{array}{l}\text { Mala gestión } \\
\text { /inexistencia del } \\
\text { indicador }\end{array}$ & $89(68)$ & $2(1)$ & $7(5)$ & $10(8)$ & $11(8)$ \\
\hline $\begin{array}{l}\text { Se aprecia de forma } \\
\text { muy modesta }\end{array}$ & 0 & $10(7)$ & $7(5)$ & $9(7)$ & $31(24)$ \\
\hline $\begin{array}{l}\text { Se aprecia de forma } \\
\text { más notable }\end{array}$ & 0 & $49(38)$ & $48(37)$ & $52(40)$ & $50(38)$ \\
\hline $\begin{array}{l}\text { Buena } \\
\text { gestión/existencia del } \\
\text { indicador }\end{array}$ & $41(31)$ & $69(54)$ & $68(53)$ & $59(45)$ & $38(30)$ \\
\hline
\end{tabular}

\subsection{Diseño de la página}

A tenor del análisis de los indicadores que se han definido para medir, tanto la sobrecarga informativa visual, como la amigabilidad de la interfaz, en general, las webs de las pequeñas y medianas empresas consiguen ofrecer la información sin cargar demasiado al usuario y a través de una interfaz sin mucho ruido visual.

El diseño de la página, desde el punto de vista de un usuario, puede considerarse correcto, lo que agiliza la lectura en pantalla, evitando que el usuario cierre la página 
nada más acceder a ella, evitando la fatiga en la búsqueda de información y la sensación de densidad informativa.

Tabla 3. Diseño de la página web. Fuente: elaboración propia

\begin{tabular}{|c|c|c|c|c|c|c|}
\hline & \multicolumn{3}{|c|}{$\begin{array}{c}\text { SOBRECARGA } \\
\text { INFORMATIVA VISUAL } \\
\end{array}$} & \multicolumn{3}{|c|}{ INTERFAZ } \\
\hline & $\begin{array}{l}\text { Tamaño } \\
\text { fuente }\end{array}$ & $\begin{array}{c}\text { Uso del } \\
\text { espacio } \\
\text { visual }\end{array}$ & $\begin{array}{l}\text { Colores y } \\
\text { tipografía }\end{array}$ & $\begin{array}{c}\text { Zonas en } \\
\text { blanco }\end{array}$ & $\begin{array}{l}\text { Efectos } \\
\text { tipográficos }\end{array}$ & $\begin{array}{l}\text { Contraste } \\
\text { fuente/fondo }\end{array}$ \\
\hline $\begin{array}{l}\text { Mala } \\
\text { gestión/inexistencia } \\
\text { del indicador }\end{array}$ & 0 & $14(11)$ & $30(23)$ & $7(5)$ & $11(8)$ & $6(5)$ \\
\hline $\begin{array}{l}\text { Se aprecia de forma } \\
\text { muy modesta }\end{array}$ & $2(1)$ & $18(14)$ & $24(18)$ & $49(38)$ & $43(33)$ & $31(24)$ \\
\hline $\begin{array}{l}\text { Se aprecia de forma } \\
\text { más notable }\end{array}$ & $70(54)$ & $43(33)$ & $33(25)$ & $22(17)$ & $30(23)$ & $55(42)$ \\
\hline $\begin{array}{l}\text { Buena } \\
\text { gestión/existencia } \\
\text { del indicador }\end{array}$ & $58(45)$ & $55(42)$ & $43(34)$ & $52(40)$ & $46(36)$ & $38(29)$ \\
\hline
\end{tabular}

\subsection{Elementos multimedia}

Tabla 4. Elementos multimedia en la web. Fuente: elaboración propia

\begin{tabular}{|c|c|c|c|c|c|}
\hline & \multirow{2}{*}{\multicolumn{4}{|c|}{ FOTOS }} & \multirow{3}{*}{$\begin{array}{l}\text { Existencia } \\
\text { de videos }\end{array}$} \\
\hline & & & & & \\
\hline & $\begin{array}{c}\text { Existencia } \\
\text { de fotos }\end{array}$ & $\begin{array}{c}\text { Bien } \\
\text { recortadas }\end{array}$ & $\begin{array}{c}\text { Contextualizadas } \\
\text { en la web }\end{array}$ & $\begin{array}{c}\text { Resolución } \\
\text { cuidada }\end{array}$ & \\
\hline $\begin{array}{l}\text { Mala gestión } \\
\text { /inexistencia del } \\
\text { indicador }\end{array}$ & $30(23)$ & $30(23)$ & $30(23)$ & $46(35)$ & $118(91)$ \\
\hline $\begin{array}{l}\text { Se aprecia de forma } \\
\text { muy modesta }\end{array}$ & 0 & $11(8)$ & $20(15)$ & $12(10)$ & 0 \\
\hline $\begin{array}{l}\text { Se aprecia de forma } \\
\text { más notable }\end{array}$ & 0 & $18(14)$ & $19(15)$ & 25 (19) & 0 \\
\hline $\begin{array}{l}\text { Buena } \\
\text { gestión/existencia } \\
\text { del indicador }\end{array}$ & $100(77)$ & $71(55)$ & $61(47)$ & $47(36)$ & $12(9)$ \\
\hline
\end{tabular}

Los elementos multimedia ofrecen unos resultados positivos, sobre todo en cuanto a las fotografías se refiere. Se puede considerar, por tanto, que los contenidos multimedia puestos a disposición de los usuarios son de carácter básico.

La mayoría de las webs (77\%) cuenta con fotografías, que ilustran los contenidos expuestos, mostrando imágenes de sus productos o servicios, y cuya función principal es la de acompañar a los contenidos textuales. En general, muestran elementos que tienen relación en el contexto de la web (es decir que son fotos relativas a la empresa tales como la sede, los productos que ofrece, etc.) pero que, en general, no aportan un valor añadido real ya que realizan una función de meros acompañantes del texto.

Por el contrario, no se le presta mucha atención a los vídeos, siendo su presencia en las webs analizadas muy baja (en tan sólo 12 de las webs analizadas se ha detectado información sobre la empresa en formato vídeo).

\subsection{Existencia de buscadores}

- Se habilita una búsqueda sencilla en la página de inicio.

- Se encuentra fácilmente accesible.

- Permite la búsqueda avanzada.

- Muestra los resultados de forma comprensible para el usuario. 
Tabla 5. Existencia de buscadores en la web. Fuente: elaboración propia

\begin{tabular}{|l|c|c|c|c|}
\cline { 2 - 5 } \multicolumn{1}{c|}{} & \multicolumn{4}{|c|}{ BUSCADOR } \\
\cline { 2 - 5 } & $\begin{array}{c}\text { Página } \\
\text { inicio }\end{array}$ & Accesibilidad & $\begin{array}{c}\text { Búsqueda } \\
\text { avanzada }\end{array}$ & $\begin{array}{l}\text { Rresultados } \\
\text { comprensibles }\end{array}$ \\
\cline { 2 - 5 } & \multicolumn{4}{|c|}{ Frecuencia (\%) } \\
\hline $\begin{array}{l}\text { Mala gestión/inexistencia } \\
\text { del indicador }\end{array}$ & $106(82)$ & $106(82)$ & $125(96)$ & $107(82)$ \\
\hline $\begin{array}{l}\text { Se aprecia de forma muy } \\
\text { modesta }\end{array}$ & 0 & $8(6)$ & 0 & 0 \\
\hline $\begin{array}{l}\text { Se aprecia de forma más } \\
\text { notable }\end{array}$ & 0 & 0 & 0 & $10(8)$ \\
\hline $\begin{array}{l}\text { Buena gestión/existencia } \\
\text { del indicador }\end{array}$ & $24(18)$ & $16(12)$ & $5(4)$ & $13(10)$ \\
\hline
\end{tabular}

La presencia de este indicador de la usabilidad es muy baja en las webs (está presente en el 18\%). Este resultado puede estar explicado por el hecho de que las PYMES entienden que todos los contenidos son fácilmente alcanzables y no ven la necesidad de guiar al usuario durante su navegación por la web.

La sencillez de estas webs, no hacen necesario la presencia de un buscador que facilite a los usuarios encontrar la información que necesitan. A pesar de esto, los usuarios que acceden a un sitio web lo hacen con el fin de encontrar algo y, en caso de no hacerlo, pueden tener una sensación de frustración y abandonar el sitio, por lo que la web debería proporcionarle la forma de encontrar lo que busque, satisfaciendo sus objetivos de búsqueda para evitar que abandone la página.

Si bien es destacable que las webs que cuentan con esta sección ofrecen los resultados de una manera comprensible, y la sitúan en un lugar adecuado, no es menos cierto que de los resultados se desprende una falta de interés por ofrecer al usuario la opción de búsqueda, lo que puede transmitir una falta de orientación al usuario.

\section{Conclusiones}

Una vez que se han presentado los principales resultados relativos a las variables de análisis que definen la usabilidad de una página web, y a modo de conclusión, se ha querido determinar el nivel de eficacia de los sites corporativos que forman parte de la muestra, considerando la categoría usabilidad.

Para ello, en primer lugar, se ha elaborado un baremo de medida en el que se han sumado los valores obtenidos para cada uno de los 25 ítems analizados (la máxima puntuación posible sería de 75 puntos) y el resultado ha sido dividido por el número total de indicadores que se han aplicado (en este caso 25). Para la obtención de un porcentaje, dicho resultado se ha multiplicado por 100.

Finalmente, en función de esa cifra, que mide la eficacia web desde el punto de vista de su usabilidad, y siguiendo la propuesta realizada por Díaz, Martín y Esteban (2008), aunque añadiendo un intervalo más (se ha añadido el intervalo "eficacia muy baja" con respecto a la propuesta de Díaz et al.) se ha considerado que:

- Una web que no alcance una puntuación de 45,75 (por debajo del 25\% de la máxima puntuación) sería una web empresarial con una muy baja eficacia a la hora de comunicarse con sus públicos.

- En segundo lugar, se considera que una web con una puntuación entre 45,75 y 91,5 puntos (entre el 25\% y el 50\% de la puntuación total) tendría una eficacia baja 
- Aquellas webs con una puntuación entre 91,5 y 137,25 puntos (entre el 50\% y el $75 \%$ ) son consideradas webs medianamente eficaces

- Por último, se considera que una web tiene una eficacia alta cuando alcanza una puntuación superior a 137,25 puntos ( $75 \%$ o más del total de la puntuación).

- A modo de resumen y considerando que la puntuación máxima a alcanzar es de 75 puntos, el baremo de medida sería el siguiente:

- Eficacia alta: $137,23-183$ puntos (75\%-100\%)

- Eficacia media: 91,5-137,23 puntos (50\%-75\%)

- Eficacia baja: $45,75-91,5$ puntos $(25 \%-50 \%)$

- Eficacia muy baja: 0-45,75 puntos (0\%-25\%)

A modo de ejemplo, una web que haya obtenido una puntuación total de 41 puntos como resultado de la suma de las valoraciones de cada uno de los ítems, tendría una puntuación porcentual final de 54,64\% sobre el máximo de puntos posibles. Por lo que se consideraría que la eficacia que esta web tiene para las PYMES a la hora de comunicarse con sus públicos sería media.

Una vez aplicado dicho baremo, los resultados se presentan en la siguiente tabla desagregados, además, por tamaño de las empresas y sector de actividad.

Tabla 6. Eficacia de las webs en función de la navegabilidad/usabilidad.

Distribución por tamaño y sector de actividad. Fuente: elaboración propia

\begin{tabular}{|l|l|c|l|l|}
\hline \multicolumn{5}{|c|}{ Distribución por categoría, usabilidad, tamaño y sector de } \\
actividad
\end{tabular}

Como conclusión, se puede afirmar que las webs de las PYMES tienen, por lo general, una usabilidad aceptable y poseen unos índices de navegabilidad adecuados (el $59 \%$ de las mismas tienen una eficacia media-alta). La desagregación atendiendo a la usabilidad de las páginas webs deja, sobre todo un resultado destacable: son 37 las webs empresariales que alcanzan una eficacia alta desde el punto de vista de la navegación de las mismas.

Disminuyen, en consecuencia, el número de webs con una eficacia media, baja y muy baja. En el caso de la eficacia muy baja, sólo 13 de las webs analizadas se sitúan en esta posición. 
Atendiendo a la distribución sectorial, las webs del sector servicios son las que ofrecen los mejores resultados. El 62\% tienen una eficacia media-alta. En este caso, el sector industria es el que ofrece las webs con mayor usabilidad, el $42 \%$ de las mismas tienen una eficacia alta en esta categoría.

Son las webs del sector construcción las que ofrecen peores resultados. De hecho, el $100 \%$ de las webs tienen una eficacia baja o muy baja. En términos generales, y considerando que en la muestra sólo hay 6 webs del sector construcción, son las empresas del sector primario las que ofrecen los peores resultados, puesto que el $31 \%$ de las webs de este sector ofrecen una usabilidad muy baja.

Estos datos han de entenderse como positivos, ya que la usabilidad es considerada un aspecto clave en la creación de una buena imagen. Las webs que se perciben como más fáciles de abrir, navegar o utilizar crean una actitud y una imagen más favorable entre los usuarios. Los usuarios que navegan con mayor facilidad por una web y encuentran la información que desean rápidamente, obtienen una mejor impresión de la empresa y satisfacen en mayor grado sus expectativas (Huertas \& Xifra, 2009).

En definitiva, la mayoría de las pequeñas y medianas empresas cumplen con los principios básicos que debe contener una página web usable por lo que la navegación no entraña dificultad alguna. Se trata de páginas fáciles de navegar, de diseño sencillo y bien estructurado. Se aprecia una claridad en la presentación, caracterizada por un buen contraste figura/fondo y una correcta legibilidad.

A pesar de esto, no se puede obviar que la mejor utilización de las variables e indicadores que se han identificado en la literatura podrían mejorar sustancialmente la eficacia de la comunicación web de las pequeñas y medianas empresas con sus públicos, porque marcan las pautas para el diseño de un site corporativo.

\section{Referencias bibliográficas}

ALAMDARI, Fariba y MASON, Keith (2006): "The future of airline distribution". Journal of Air Transport Management, vol. 12, num. 3, pp. 122-134.

DOI: http://dx.doi.org/10.1016/j.jairtraman.2005.11.005

BAUTISTA, Laura (2012): "Los cambios en la web 2.0: una nueva sociabilidad". Estudios sobre el Mensaje Periodístico, vol. 18, núm. especial octubre, pp. 121-128. Madrid, Servicio de Publicaciones de la Universidad Complutense.

CASTILLO, Ana; CARRILLO, M ${ }^{a}$ Victoria; y LUNA, Ma Rosario (2012): "Estudio de las sedes webs de las PYMES mexicanas como herramienta de apoyo a sus estrategias de comunicación empresarial", en http://www.ae-ic.org/tarragona2012/contents/comunicacions_cd/ok/63.pdf

DA SILVA, Rui Vinhas and ALWI, Sharifa (2006): "Online corporate brand image, satisfaction and loyalty". Brand management, vol. 16, pp. 119-144. DOI: http://dx.doi.org/10.1057/palgrave.bm.2550137

DÍAZ, Estrella; MARTÍN, David; y ESTEBAN, Águeda (2008): "Evaluación de la eficacia de las páginas web: un análisis de contenido de las principales compañías aéreas", en DE CASTRO, Emilio y DÍAZ, Francisco: Universidad, Sociedad y Mercados Globales. Asociación Europea de Dirección y Economía de empresa. 
GARCÍA, María (2012): "La comunicación de la marca en las pymes a través de sus sedes webs. El caso de las pymes extremeñas. El caso de las PYMES extremeñas". Tesis doctoral. Universidad de Extremadura.

GARCÍA, María y CASTILLO, Ana (2010): "Webs usables y accesibles en PYMES. Retos para el futuro". Revista Latina de Comunicación Social, vol. 65, pp. 392-409.

GARCÍA, María; CARRILLO, Ma Victoria; y CASTILLO, Ana (2012): "La usabilidad en los portales webs de las PYMES. Herramientas y usos para la comunicación empresarial". Estudios sobre el Mensaje Periodístico, vol. 18, núm. especial Octubre, pp. 365-375. Madrid, Servicio de Publicaciones de la Universidad Complutense.

GARCÍA, María; CASTILLO, Ana; y CARRILLO, Ma Victoria (2012): "Online corporate communication: SMEs and differences across sectors". Journal of Organisational Transformation \& Social Change, vol. 9, num. 3, pp. 285-300. DOI: http://dx.doi.org/10.1386/jots.9.3.285_1

GIBSON, Dirk (2000): "The cyber-revolution in product recall public relations". Public Relations Quarterly, vol. 45, num. 2, pp. 24-26.

GÓMEZ, Begoña; TAPIA, Alejandro; y DÍAZ, Óscar (2012): "La Comunicación corporativa a través de las páginas web: el caso de las ONGS españolas". Revista de Comunicación Vivat Academia, Vol. XIV, núm.. 120, pp. 1-20.

HA, Myounghee \& LOVE, Curtis (2005): "Exploring content and design factors associated with convention and visitors bureau web site development: an analysis of recognition by meeting planners". Journal of Convention and Event Tourism, vol. 7, num. 1, pp. 43-59.

HASSAN, Yusef (2006): "Factores del diseño web orientado a la satisfacción y no frustración de uso". Revista Española de Documentación Científica, vol. 24, núm. 1, pp. 239-257.

HERNÁNDEZ, Blanca; JIMÉNEZ, Julio; y MARTÍN, Ma José (2010): “Efecto de la calidad de un sitio web sobre el tráfico recibido. El caso de la banca electrónica en España”. Information Research, vol. 15, num. 2, paper 429.

HUERTAS, Assumpció y XIFRA, Jordi (2009): “Marcas o genéricos? La Comunicación en línea de las marcas farmacéuticas". Zer. Revista de Estudios de Comunicación, vol. 14, núm. 27, pp. 251-270.

JULIÁ, Juan Francisco; GARCÍA, Gabriel; y POLO, Fernando (2004): "La información divulgada a través de Internet por las cooperativas". CIRIEC-España, Revista de Economía Pública, Social y Cooperativa, vol. 49, pp. 167-192.

KAPLANIDOU, Kyriaki and VOGT, Christine (2006): "A Structural Analysis of Destination Travel Intentions as a Function of Website features". Journal of Travel Research, vol. 45, num. 2, pp. 204-216.

KLINE, Sheryl; MORRISON, Alastair; y JOHN, Andrew (2004): "Exploring bed and breakfast web sites: a balanced scorecard approach". Journal of travel and tourism Marketing, vol. 17, num. 2, pp. 253-267. 
KRUG, Steve (2006): No me hagas pensar. Una aproximación a la usabilidad en la web. Madrid, Pearson Educación.

LI, Dahui; BROWNE, Glenn; y CHAU, Patrick (2006): “An empirical investigation of web site use using a commitment-based model". Decision Sciences, vol. 37, num. 3, pp. 196-211.

MORENO, Ángeles y CAPRIOTTI, Paul (2006): “La comunicación de las empresas españolas en sus webs corporativas. Análisis de la información de responsabilidad social, ciudadanía corporativa y desarrollo sostenible". Zer, vol. 21, pp. 47-62.

MORRISON, Alastair, TAYLOR, Stephen y DOUGLAS, Alecia (2004): "Web site evaluation in tourims and hospitality: the art is not yet stated". Journal of travel and tourism Marketing, vol. 17, num. 2/3, pp. 233-251.

MILOJEVIC, Ana; KLEUT, Jelena; y NINKOVIC, Danka (2013): "Propuestas metodológicas para el estudio de la interactividad en revistas de comunicación". Comunicar, núm. 41, pp. 93-103.

NIELSEN, Jakob (1993): Usability engineering. San Francisco, Morgan Kaufmann.

NIELSEN, Jakob y TAHIR, Marie (2002): Usabilidad de páginas de inicio: análisis de 50 sitios web. Madrid, Pearson Educación.

PALAZÓN, Alfonso (2001): "Comunicación web: el valor de los contenidos de la Red”. Comunicar, núm. 17, pp. 93-96.

PALMER, Jonathan (2002): "Web site usability, design and performance metrics". Information System Research, vol. 13, num. 2, pp. 151-167. DOI: http://dx.doi.org/10.1287/isre.13.2.151.88

PIÑUEL, José Luis (2002): "Epistemología, metodología y técnicas de análisis de contenido". Estudios de Sociolingüística, núm. 3, pp. 1-42.

POLLACH, Irene (2005): "Corporate self-presentation on the WWW. Strategies for enhancing usability, credibility and utility". Corporate Communications: An international Review, vol. 10, num. 4, pp. 285-301.

ROS, Vicente (2008): E-branding. Posiciona tu marca en la Red. Oleiros (A Coruña), Netbiblo.

SUTCLIFFE, Alistair (2002): "Assessing the Reliability of Heuristic Evaluation for Website Attractiveness and Usability". Proceedings of the 35th Hawaii International Conference on System Sciences, Hawaii.

VILLENA, Eduardo (2013): La gestión de la Comunicación en las empresas de moda española. Tesis doctoral. Universidad de Málaga.

WANG, Youcheng and FESENMAIER, Daniel (2006): "Identifying the success factors of web-based marketing strategy: an investigation of convention and visitors bureaus in the United States". Journal of Travel Research, vol. 44, num. 3, pp. 239-249. 
XING, Yuan and GRANDT, David (2006): "Developing a framework for measuring physical distribution service quality of multi-channel and pure player Internet retailers". International Journal of retail and Distribution Management, vol. 34, num. 4/5, pp. 278-289.

YOON, Moon; YOUNG, Duk; y WON, Tae (2006): "Impact of e-business on air travel markets: Distributions of airline tickets in Korea". Journal of Air Transport Management, vol. 12, num. 5, pp. 31-46. DOI: http://dx.doi.org/10.1016/j.jairtraman.2006.07.002.

Pedro Pablo Marín Dueñas es investigador postdoctoral en la Universidad de Cádiz.

Carmen Lasso de la Vega González es Profesora Contratada Doctora. Universidad de Cádiz.

Juan José Mier-Terán Franco es Profesor Titular. Universidad de Cádiz. 